\title{
On the convergence rates in the asymmetric SLLN for independent and nonidentically distributed random fields
}

Mi Hwa Ko*

\section{*Correspondence:}

songhack@wonkwang.ac.kr

Division of Mathematics and

Informational Statistics, Wonkwang

University, Jeonbuk, 570-749, Korea

\begin{abstract}
The purpose of this paper is to obtain the convergence rates in strong laws of large numbers for nonidentically distributed and independent random fields such that different indices have different powers in the normalization.

MSC: $60 \mathrm{~F} 05 ; 62 \mathrm{~F} 15$

Keywords: asymmetric; convergence rate; nonidentical distribution; random field; strong law of large numbers
\end{abstract}

\section{Introduction}

Let $Z_{+}^{d}$, where $d$ is a positive integer, denote positive integer $d$-dimensional lattice points. For the elements of $Z_{+}^{d}$ we use bold symbols $\mathbf{m}, \mathbf{n}$ and so forth. Further, we will assume the usual partial ordering for elements of $Z_{+}^{d}$, i.e. for $\mathbf{m}=\left(m_{1}, m_{2}, \ldots, m_{d}\right)$ and $\mathbf{n}=\left(n_{1}, n_{2}, \ldots, n_{d}\right), \mathbf{m} \leq \mathbf{n}$ if and only if $m_{i} \leq n_{i}$ for all $i=1,2, \ldots, d$. The strict inequality $\mathbf{m}<\mathbf{n}$ is defined as follows: $\mathbf{m}<\mathbf{n}$ if and only if $\mathbf{m} \leq \mathbf{n}$ and $\mathbf{m} \neq \mathbf{n}$. We assume that $\mathbf{n} \rightarrow \infty$ means $\max _{1 \leq i \leq d} n_{i} \rightarrow \infty$. We also use $|\mathbf{n}|$ for $\prod_{i=1}^{d} n_{i}$.

Most of the results concerning limit theorems, especially for random fields, are obtained for identically distributed random variables.

Kuczmaszewska and Łagodowski [1] considered the concept of weak boundedness as follows.

Definition 1.1 (Kuczmaszewska and Łagodowski [1]) The random variables $\left\{X_{\mathbf{k}}, \mathbf{k} \in Z_{+}^{d}\right\}$ are said to be weakly bounded by the random variable $\xi$ if there exist some constants $c_{1}, c_{2}>0, \mathbf{n}_{\mathbf{0}} \in Z_{+}^{d}$ and $x_{0}>0$ such that for every $x>x_{0}$ and $\mathbf{n} \geq \mathbf{n}_{\mathbf{0}}, \mathbf{n} \in Z_{+}^{d}$

$$
c_{1} P(|\xi|>x) \leq \frac{1}{|\mathbf{n}|} \sum_{\mathbf{k} \leq \mathbf{n}} P\left(\left|X_{\mathbf{k}}\right|>x\right) \leq c_{2} P(|\xi|>x) .
$$

Clearly, the regular cover implies weak boundedness. If only the right-hand side inequality is satisfied we say that the random field $\left\{X_{\mathbf{n}}, \mathbf{n} \in Z_{+}^{d}\right\}$ and the random variable $\xi$ satisfy the weak mean dominating condition.

@2014 Ko; licensee Springer. This is an Open Access article distributed under the terms of the Creative Commons Attribution License (http://creativecommons.org/licenses/by/2.0), which permits unrestricted use, distribution, and reproduction in any medium, provided the original work is properly cited. 
Gut [2] proved Marcinkiewicz laws and convergence rates in the law of large numbers for i.i.d. random fields and Gut [3] also showed convergence rates for probabilities of moderate deviations for independent random fields.

Recently, the limit theorems for independent random fields such that different indices have different powers in the normalization are investigated. For example, Thanh [4] provided the strong law of large numbers for independent random fields and Gut and Stadtmüller [5] proved the Marcinkiewicz-Zygmund law for i.i.d. random fields and Gut and Stadtmüller [6] also obtained the Hsu-Robbins-Erdős-Spitzer-Baum-Katz theorem for i.i.d. random fields.

It is clear that independent random fields imply martingale difference random fields. Hence, from Theorem 4.1 in Kuczmaszewska and Łagodowski [1] we obtained the following convergence rates in the strong law of large numbers for independent random fields with nonidentical distribution.

Theorem 1.2 Let $\left\{X_{\mathbf{n}}, \mathbf{n} \in Z_{+}^{d}\right\}$ be the a field of independent random variables with $E X_{\mathbf{n}}=0$ for $\mathbf{n} \in Z_{+}^{d}$. Assume, for $r>1, \alpha>\frac{1}{2}$ and $\alpha r>1$,

$$
\begin{aligned}
& \sum_{\mathbf{n}}|\mathbf{n}|^{\alpha r-2} \sum_{\mathbf{i} \leq \mathbf{n}} P\left\{\left|X_{\mathbf{i}}\right|>|\mathbf{n}|^{\alpha}\right\}<\infty, \\
& \sum_{\mathbf{n}}|\mathbf{n}|^{\alpha(r-2)-2} \sum_{\mathbf{i} \leq \mathbf{n}} E\left(\left|X_{\mathbf{i}}\right|^{2} I\left[\left|X_{\mathbf{i}}\right| \leq|\mathbf{n}|^{\alpha}\right]\right)<\infty,
\end{aligned}
$$

and

$$
\sum_{\mathbf{n}}|\mathbf{n}|^{\alpha r-2} P\left\{\max _{\mathbf{j} \leq \mathbf{n}}\left|\sum_{\mathbf{i} \leq \mathbf{j}} E\left(X_{\mathbf{i}} I\left[\left|X_{\mathbf{i}}\right| \leq|\mathbf{n}|^{\alpha}\right]\right)\right|>\epsilon|\mathbf{n}|^{\alpha}\right\}<\infty
$$

for all $\epsilon>0$. Then

$$
\sum_{\mathbf{n}}|\mathbf{n}|^{\alpha r-2} P\left\{\max _{\mathbf{j} \leq \mathbf{n}}\left|S_{\mathbf{j}}\right|>\epsilon|\mathbf{n}|^{\alpha}\right\}<\infty \quad \text { for all } \epsilon>0,
$$

where $S_{\mathbf{j}}=\sum_{\mathbf{i} \leq \mathbf{j}} X_{\mathbf{i}}$.

From Corollary 4.1 in Kuczmaszewska and Łagodowski [1] we also obtain the following corollary.

Corollary 1.3 Let $\left\{X_{\mathbf{n}}, \mathbf{n} \in Z_{+}^{d}\right\}$ be a field of independent random variables with $E X_{\mathbf{n}}=0$ for $\mathbf{n} \in Z_{+}^{d}$. Then (1.2), (1.3), and

$$
\frac{1}{|\mathbf{n}|^{\alpha}} \max _{\mathbf{j} \leq \mathbf{n}} \sum_{\mathbf{i} \leq \mathbf{j}} E\left(X_{\mathbf{i}} I\left[\left|X_{\mathbf{i}}\right| \leq|\mathbf{n}|^{\alpha}\right]\right) \rightarrow 0 \quad \text { as }|\mathbf{n}| \rightarrow \infty
$$

imply (1.5).

Kuczmaszewska and Łagodowski [1] investigated the following convergence rate for independent random field weakly dominated by the random variable $\xi$. 
Theorem 1.4 (Kuczmaszewska and Łagodowski [1]) Let $\left\{X_{\mathbf{n}}, \mathbf{n} \in Z_{+}^{d}\right\}$ be a field of independent random variables weakly dominated by the random variable $\xi$ and $E X_{\mathbf{n}}=0$ for $\mathbf{n} \in Z_{+}^{d}$. Moreover, we assume that for $1<r<2,1 \leq p \leq d$

$$
E|\xi|^{r}\left(\log ^{+}|\xi|\right)^{p-1}<\infty
$$

where $\log x^{+}=\max \{1, \log x\}$. Then, for $\alpha r>1$ and $\alpha>\frac{1}{2}$, (1.5) holds.

In this paper we generalize the above results (Theorem 1.2, Corollary 1.3, and Theorem 1.4) to the case where different indices have different powers in the normalization.

\section{Results}

In Section 1 every coordinate $n_{k}, k=1,2, \ldots, d$, is raised to the same power $\alpha$. The main point of this section is to allow for different powers for different coordinates. In order to continue we therefore define $\boldsymbol{\alpha}=\left(\alpha_{1}, \alpha_{2}, \ldots, \alpha_{d}\right)$, where, w.l.o.g., we assume that the coordinates are arranged in nondecreasing order, such that $\alpha_{1}$ is the smallest one and $\alpha_{d}$ the largest one. We further let $p$ denote the number of $\alpha$ 's which are equal to the smallest one, that is, $p=\max \left\{k: \alpha_{k}=\alpha_{1}\right\}$.

As is easily seen the domain of interest concerning the $\alpha$ 's becomes

$$
\frac{1}{2} \leq \alpha_{1} \leq \alpha_{2} \leq \cdots \leq \alpha_{d} \leq 1
$$

where the boundary $\frac{1}{2}$ takes us into the realm of the central limit theorem and the boundary 1 corresponds to the Kolmogorov strong law.

For ease of notation, we use the notation $\mathbf{n}^{\alpha}=\left(n_{1}^{\alpha_{1}}, n_{2}^{\alpha_{2}}, \ldots, n_{d}^{\alpha_{d}}\right)$ and $\left|\mathbf{n}^{\alpha}\right|=\prod_{i=1}^{d} n_{i}^{\alpha_{i}}$.

Lemma 2.1 (Gut and Stadtmüller [6]) Let $\xi$ be a random variable. Let $\alpha_{1}>\frac{1}{2}$ and $1 \leq p \leq$ $d$, where $\alpha=\left(\alpha_{1}, \alpha_{2}, \ldots, \alpha_{d}\right)$ with $\frac{1}{2}<\alpha_{1}=\cdots=\alpha_{p}<\alpha_{p+1} \leq \cdots \leq \alpha_{d} \leq 1$. Then, for any $r>0$ and all $\epsilon>0$,

$$
E|\xi|^{r}\left(\log ^{+}|\xi|\right)^{p-1}<\infty \quad \Leftrightarrow \quad \sum_{\mathbf{n}}|\mathbf{n}|^{\alpha_{1} r-1} P\left(|\xi|>\epsilon\left|\mathbf{n}^{\alpha}\right|\right)<\infty
$$

The following lemma appeared in Lemma 3.1 of Thanh [4].

Lemma 2.2 (Thanh [4]) Let $\left\{X_{\mathbf{n}}, \mathbf{n} \in Z_{+}^{d}\right\}$ be a field of independent random variables with $E X_{\mathbf{n}}=0$. Then there exists a constant $C$ depending only on $p$ and $d$ such that

$$
E\left(\max _{\mathbf{k} \leq \mathbf{n}}\left|S_{\mathbf{k}}\right|^{p}\right) \leq C \sum_{\mathbf{i} \leq \mathbf{n}} E\left|X_{\mathbf{i}}\right|^{p} \quad \text { for all } 0<p \leq 2
$$

Remark In the case $0<p \leq 1$, the independent hypothesis and the hypothesis that $E X_{\mathbf{n}}=$ 0 are superfluous, and $C$ is given by $C=1$. In the case $1<p<2, C$ is given by $C=2\left(\frac{p}{p-1}\right)^{p d}$. In the case $p=2$, Lemma 2.2 was proved by Wichura [7] and $C$ is given by $C=4^{d}$.

Theorem 2.3 Let $\left\{X_{\mathbf{n}}, \mathbf{n} \in Z_{+}^{d}\right\}$ be a field of independent random variables with $E X_{\mathbf{n}}=0$ for $\mathbf{n} \in Z_{+}^{d}$ and let $\alpha=\left(\alpha_{1}, \alpha_{2}, \ldots, \alpha_{d}\right)$, where $\frac{1}{2}<\alpha_{1}=\cdots=\alpha_{p}<\alpha_{p+1} \leq \cdots \leq \alpha_{d} \leq 1$ and 
$1 \leq p \leq d$. Assume, for $r>1$ and $\alpha_{1} r>1$,

$$
\begin{aligned}
& \sum_{\mathbf{n}}|\mathbf{n}|^{\alpha_{1} r-2} \sum_{\mathbf{i} \leq \mathbf{n}} P\left(\left|X_{\mathbf{i}}\right|>\left|\mathbf{n}^{\alpha}\right|\right)<\infty, \\
& \sum_{\mathbf{n}}|\mathbf{n}|^{\alpha_{1}(r-2)-2} \sum_{\mathbf{i} \leq \mathbf{n}} E\left(\left|X_{\mathbf{i}}\right|^{2} I\left(\left|X_{\mathbf{i}}\right| \leq\left|\mathbf{n}^{\alpha}\right|\right)\right)<\infty,
\end{aligned}
$$

and

$$
\sum_{\mathbf{n}}|\mathbf{n}|^{\alpha_{1} r-2} P\left\{\max _{\mathbf{j} \leq \mathbf{n}}\left|\sum_{\mathbf{i} \leq \mathbf{j}} E\left(X_{\mathbf{i}} I\left[\left|X_{\mathbf{i}}\right| \leq\left|\mathbf{n}^{\alpha}\right|\right]\right)\right|>\epsilon\left|\mathbf{n}^{\alpha}\right|\right\}<\infty
$$

for all $\epsilon>0$. Then we obtain

$$
\sum_{\mathbf{n}}|\mathbf{n}|^{\alpha_{1} r-2} P\left\{\max _{\mathbf{j} \leq \mathbf{n}}\left|S_{\mathbf{j}}\right|>\epsilon\left|\mathbf{n}^{\alpha}\right|\right\}<\infty \quad \text { for all } \epsilon>0
$$

Proof Let $X_{\mathbf{n}, \mathbf{i}}=X_{\mathbf{i}} I\left[\left|X_{\mathbf{i}}\right| \leq\left|\mathbf{n}^{\alpha}\right|\right], X_{\mathbf{n}, \mathbf{i}}^{*}=X_{\mathbf{n}, \mathbf{i}}-E\left(X_{\mathbf{n}, \mathbf{i}}\right)$ and $S_{\mathbf{n}, \mathbf{j}}^{*}=\sum_{\mathbf{i} \leq \mathbf{j}} X_{\mathbf{n}, \mathbf{i}}^{*}$. Then

$$
\begin{aligned}
\sum_{\mathbf{n}}|\mathbf{n}|^{\alpha_{1} r-2} P\left\{\max _{\mathbf{j} \leq \mathbf{n}}\left|S_{\mathbf{j}}\right|>\epsilon\left|\mathbf{n}^{\alpha}\right|\right\} \\
\leq \sum_{\mathbf{n}}|\mathbf{n}|^{\alpha_{1} r-2} \sum_{\mathbf{i} \leq \mathbf{n}} P\left(\left|X_{\mathbf{i}}\right|>\left|\mathbf{n}^{\alpha}\right|\right) \\
\quad+\sum_{\mathbf{n}}|\mathbf{n}|^{\alpha_{1} r-2} P\left\{\max _{\mathbf{j} \leq \mathbf{n}}\left|\sum_{\mathbf{i} \leq \mathbf{j}} X_{\mathbf{i}} I\left[\left|X_{\mathbf{i}}\right| \leq\left|\mathbf{n}^{\alpha}\right|\right]\right|>\epsilon\left|\mathbf{n}^{\alpha}\right|\right\} \\
\leq \sum_{\mathbf{n}}|\mathbf{n}|^{\alpha_{1} r-2} \sum_{\mathbf{i} \leq \mathbf{n}} P\left(\left|X_{\mathbf{i}}\right|>\left|\mathbf{n}^{\alpha}\right|\right)+\sum_{\mathbf{n}}|\mathbf{n}|^{\alpha_{1} r-2} P\left\{\max _{\mathbf{j} \leq \mathbf{n}}\left|S_{\mathbf{n}, \mathbf{j}}^{*}\right|>\frac{\epsilon}{2}\left|\mathbf{n}^{\alpha}\right|\right\} \\
\quad+\sum_{\mathbf{n}}|\mathbf{n}|^{\alpha_{1} r-2} P\left\{\max _{\mathbf{j} \leq \mathbf{n}}\left|\sum_{\mathbf{i} \leq \mathbf{j}} E\left(X_{\mathbf{i}} I\left[\left|X_{\mathbf{i}}\right| \leq\left|\mathbf{n}^{\alpha}\right|\right]\right)\right|>\frac{\epsilon}{2}\left|\mathbf{n}^{\alpha}\right|\right\} \\
=I_{1}+I_{2}+I_{3} .
\end{aligned}
$$

It follows from (2.2) and (2.4) that

$$
I_{1}<\infty \text { and } I_{3}<\infty
$$

hold. It remains to prove that $I_{2}<\infty$.

By the Markov inequality and Lemma 2.2, for some positive constant $C$ we obtain

$$
\begin{aligned}
P\left\{\max _{\mathbf{j} \leq \mathbf{n}}\left|S_{\mathbf{n}, \mathbf{j}}^{*}\right|>\frac{\epsilon}{2}\left|\mathbf{n}^{\alpha}\right|\right\} & \leq C \frac{E\left(\max _{\mathbf{j} \leq \mathbf{n}} S_{\mathbf{n}, \mathbf{j}}^{*}\right)}{\left|\mathbf{n}^{\alpha}\right|^{2}} \\
& \leq C \frac{\sum_{\mathbf{i} \leq \mathbf{n}} E X_{\mathbf{n}, \mathbf{i}}^{*}}{\left|\mathbf{n}^{\alpha}\right|^{2}} \\
& \leq C\left|\mathbf{n}^{\alpha}\right|^{-2} \sum_{\mathbf{i} \leq \mathbf{n}} E\left(X_{\mathbf{i}}^{2} I\left[\left|X_{\mathbf{i}}\right| \leq\left|\mathbf{n}^{\alpha}\right|\right]\right) \\
& \leq C|\mathbf{n}|^{-2 \alpha_{1}} \sum_{\mathbf{i} \leq \mathbf{n}} E\left(X_{\mathbf{i}}^{2} I\left[\left|X_{\mathbf{i}}\right| \leq\left|\mathbf{n}^{\alpha}\right|\right]\right)
\end{aligned}
$$


since $|\mathbf{n}|^{2 \alpha_{1}} \leq\left|\mathbf{n}^{\alpha}\right|^{2}$. Therefore, by (2.3) and (2.8) we obtain

$$
\begin{aligned}
I_{2} & =\sum_{\mathbf{n}}|\mathbf{n}|^{\alpha_{1} r-2} P\left\{\max _{\mathbf{j} \leq \mathbf{n}}\left|S_{\mathbf{n}, \mathbf{j}}^{*}\right|>\frac{\epsilon}{2}\left|\mathbf{n}^{\alpha}\right|\right\} \\
& \leq C \sum_{\mathbf{n}}|\mathbf{n}|^{\alpha_{1}(r-2)-2} E\left(X_{\mathbf{i}}^{2} I\left[\left|X_{\mathbf{i}}\right| \leq\left|\mathbf{n}^{\alpha}\right|\right]\right)<\infty .
\end{aligned}
$$

Hence, by (2.6), (2.7), and (2.9) the result (2.5) follows.

Remark Theorem 2.3 generalizes Theorem 1.2 to the case where different indices have different powers in the normalization. That is, Theorem 1.2 is a special case of Theorem 2.3 with $\alpha_{1}=\alpha_{2}=\cdots=\alpha_{d}$.

Corollary 2.4 Let $\left\{X_{\mathbf{n}}, \mathbf{n} \in Z_{+}^{d}\right\}$ be a field of independent random variables such that $E X_{\mathbf{n}}=$ 0 for $\mathbf{n} \in Z_{+}^{d}$. Let $r \geq 1, \alpha_{1}>\frac{1}{2}$ and $\alpha_{1} r>1$, where $\alpha=\left(\alpha_{1}, \alpha_{2}, \ldots, \alpha_{d}\right)$ with $\frac{1}{2}<\alpha_{1}=\cdots=\alpha_{p}<$ $\alpha_{p+1} \leq \cdots \leq \alpha_{d} \leq 1$ and $1 \leq p \leq d$. Assume (2.2), (2.3), and

$$
\frac{1}{|\mathbf{n}|^{\alpha_{1}}} \max _{\mathbf{j} \leq \mathbf{n}} \sum_{\mathbf{i} \leq \mathbf{j}} E\left(X_{\mathbf{i}} I\left[\left|X_{\mathbf{i}}\right| \leq\left|\mathbf{n}^{\alpha}\right|\right]\right) \rightarrow 0 \quad \text { as }|\mathbf{n}| \rightarrow \infty
$$

Then (2.5) holds.

Proof It is easy to see that (2.10) implies (2.4). Thus, by Theorem 2.3 we get (2.5).

Remark The following corollary shows that the assumption (2.4) is natural and it reduces to the known one.

In the case of a weakly dominated random field, we have the following theorem.

Corollary 2.5 Let $\left\{X_{\mathbf{n}}, \mathbf{n} \in Z_{+}^{d}\right\}$ be a field of independent random variables weakly dominated by the random variable $\xi$ and such that $E X_{\mathbf{n}}=0$ for $\mathbf{n} \in Z_{+}^{d}$. Let $\frac{1}{2}<\alpha_{1}=\cdots=\alpha_{p}<$ $\alpha_{p+1} \leq \cdots \leq \alpha_{d} \leq 1$, where $\alpha=\left(\alpha_{1}, \alpha_{2}, \ldots, \alpha_{d}\right)$ and $1 \leq p \leq d$. Assume that for $r>2$

$$
E|\xi|^{r}\left(\log ^{+}|\xi|\right)^{p-1}<\infty
$$

Then (2.5) holds.

Proof We are going to prove Corollary 2.5 by using Corollary 2.4. It follows from Lemma 2.1 and (2.11) that, for $\alpha_{1} r>1$, we have

$$
\begin{aligned}
\sum_{\mathbf{n}}|\mathbf{n}|^{\alpha_{1} r-2} \sum_{\mathbf{i} \leq \mathbf{n}} P\left(\left|X_{\mathbf{i}}\right|>\left|\mathbf{n}^{\alpha}\right|\right) & <C \sum_{\mathbf{n}}|\mathbf{n}|^{\alpha_{1} r-1} P\left(|\xi|>\left|\mathbf{n}^{\alpha}\right|\right) \\
& =C E|\xi|^{r}\left(\log ^{+}|\xi|\right)^{p-1}<\infty,
\end{aligned}
$$

which yields (2.2). 
Furthermore, we get

$$
\begin{aligned}
\sum_{n}|\mathbf{n}|^{\alpha_{1}(r-2)-2} \sum_{\mathbf{i} \leq \mathbf{n}} E X_{\mathbf{i}}^{2} I\left[\left|X_{\mathbf{i}}\right| \leq\left|\mathbf{n}^{\alpha}\right|\right] \\
\leq \sum_{\mathbf{n}}|\mathbf{n}|^{\alpha_{1}(r-2)-2}\left(|\mathbf{n}| E \xi^{2} I\left[|\xi| \leq\left|\mathbf{n}^{\alpha}\right|\right]\right. \\
\left.\quad+|\mathbf{n}|^{1+2 \alpha_{1}} P\left(|\xi|>\left|\mathbf{n}^{\alpha}\right|\right)\right) \\
=C \sum_{\mathbf{n}}|\mathbf{n}|^{\alpha_{1}(r-2)-1} E \xi^{2} I\left[|\xi| \leq\left|\mathbf{n}^{\alpha}\right|\right] \\
\quad+C \sum_{\mathbf{n}}|\mathbf{n}|^{\alpha_{1} r-1} P\left(|\xi|>\left|\mathbf{n}^{\alpha}\right|\right) \\
=I_{4}+I_{5} .
\end{aligned}
$$

It follows from (2.11) and Lemma 2.1 that

$$
I_{5}<\infty
$$

Let $\frac{1}{2}<\alpha_{1}=\cdots=\alpha_{p}<\alpha_{p+1} \leq \cdots \leq \alpha_{d} \leq 1$ and let $v=n_{1} \times \cdots \times n_{p}$.

To show $I_{4}<\infty$ define

$$
f(v)=\sum_{n_{1} \cdots n_{p} \leq \nu} 1 \sim \frac{v(\log v)^{p-1}}{(p-1) !} \text { as } v \rightarrow \infty
$$

with differences $\Delta f(v)=f(v)-f(v-1)=\sum_{n_{1} \cdots n_{p}=v} 1$.

(See Gut and Stadtmüller [5].)

Then we have

$$
\begin{aligned}
& I_{4}=\sum_{\mathbf{n}}|\mathbf{n}|^{\alpha_{1}(r-2)-1} E|\xi|^{2} I\left[|\xi| \leq\left|\mathbf{n}^{\alpha}\right|\right] \\
& =\sum_{v, n_{p+1}, \ldots, n_{d}=1}^{\infty} \Delta f(v)\left(v \cdot n_{p+1} \cdots n_{d}\right)^{\alpha_{1}(r-2)-1} \sum_{j=1}^{v^{\alpha_{1}} n_{p+1}^{\alpha_{p+1} \cdots n_{d}}} E\left(\xi^{\alpha_{d}} I(j-1<|\xi| \leq j)\right) \\
& \leq \sum_{v, n_{p+1}, \ldots, n_{d}=1}^{\infty}\left(v \cdot n_{p+1} \cdots n_{d}\right)^{\alpha_{1}(r-2)-1} \Delta f(v) \sum_{j=1}^{\nu^{\alpha_{1}} \sum_{p+1}^{\alpha_{p+1} \ldots n_{d}}} j^{\alpha_{d}} P(j-1<|\xi| \leq j) \\
& \leq C \sum_{v, n_{p+1}, \ldots, n_{d}=1}^{\infty}\left(v \cdot n_{p+1} \cdots n_{d}\right)^{\alpha_{1}(r-2)-1} \Delta f(v) \sum_{j=1}^{v^{\alpha_{1}} n_{p+1}^{\alpha_{p+1} \cdots n_{d}}}\left(\sum_{i=1}^{j} i\right) P(j-1<|\xi| \leq j) \\
& \leq C \sum_{\nu, n_{p+1}, \ldots, n_{d}=1}^{\infty}\left(v \cdot n_{p+1} \cdots n_{d}\right)^{\alpha_{1}(r-2)-1} \Delta f(v) \sum_{j=1}^{\nu^{\alpha_{1}} n_{p+1}^{\alpha_{p+1} \ldots n_{d}}} j P(|\xi|>j) \\
& \leq C \sum_{n_{p+1}, \ldots, n_{d}=1}^{\infty}\left(n_{p+1} \cdots n_{d}\right)^{\alpha_{1}(r-2)-1}\left\{\sum_{j=1}^{\substack{\alpha_{p+1} \ldots n_{d} \\
n_{p+1} \cdots n_{d}}} j^{r-1} j^{2-r} P(|\xi|>j) \sum_{\nu=1}^{\infty} v^{\alpha_{1}(r-2)-1} \Delta f(\nu)\right.
\end{aligned}
$$




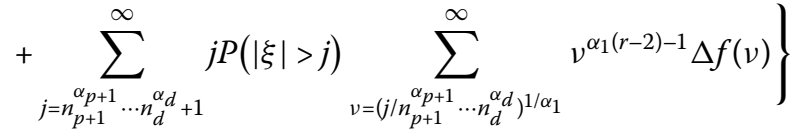

$$
\begin{aligned}
& \leq C \sum_{n_{p+1}, \ldots, n_{d}=1}^{\infty}\left(n_{p+1} \cdots n_{d}\right)^{\alpha_{1}(r-2)-1}\left\{\left(n_{p+1}^{\alpha_{p+1}} \cdots n_{d}^{\alpha_{d}}\right)^{2-r}\right. \\
& \times \sum_{j=1}^{\substack{\alpha_{p+1} \ldots n_{d} \\
n_{p+1} \cdots n_{d}}} j^{r-1} P(|\xi|>j) \sum_{v=1}^{\infty}\left(v^{\alpha_{1}(r-2)-1}-(v+1)^{\alpha_{1}(r-2)-1}\right) f(v) \\
& \left.+\sum_{\substack{\alpha_{p+1} \ldots n_{p+1}^{\alpha_{d}} \\
j=1}}^{\infty} j P(|\xi|>j) \sum_{\substack{\left.\alpha_{p+1} \\
\alpha_{p+1} \ldots n_{d}^{\alpha_{p+1}} \alpha_{d}\right)^{1 / \alpha_{1}}}}^{\infty}\left(v^{\alpha_{1}(r-2)-1}-(v+1)^{\alpha_{1}(r-2)-1}\right) f(v)\right\} \\
& \leq C \sum_{n_{p+1}, \ldots, n_{d}=1}^{\infty} n_{p+1}^{(r-2)\left(\alpha_{1}-\alpha_{p+1}\right)-1} \cdots n_{n_{d}}^{(r-2)\left(\alpha_{1}-\alpha_{d}\right)-1} \sum_{j=1}^{\infty} j^{r-1}(\log j)^{p-1} P(|\xi|>j) \\
& <\infty \text { by (2.11). }
\end{aligned}
$$

Thus, by (2.13), (2.14), and (2.15) we established (2.3).

It remains to obtain (2.10). Since $E X_{\mathbf{n}}=0$, we get

$$
\begin{aligned}
& \frac{1}{|\mathbf{n}|^{\alpha_{1}}} \max _{\mathbf{j} \leq \mathbf{n}}\left|\sum_{\mathbf{i} \leq \mathbf{j}} E\left(X_{\mathbf{i}} I\left[\left|X_{\mathbf{i}}\right| \leq\left|\mathbf{n}^{\alpha}\right|\right]\right)\right| \\
& \quad=\frac{1}{|\mathbf{n}|^{\alpha_{1}}} \max _{\mathbf{j} \leq \mathbf{n}}\left|\sum_{\mathbf{i} \leq \mathbf{j}} E\left(X_{\mathbf{i}} I\left[\left|X_{\mathbf{i}}\right|>\left|\mathbf{n}^{\alpha}\right|\right]\right)\right| \\
& \quad \leq \frac{1}{|\mathbf{n}|^{\alpha_{1}}} \sum_{\mathbf{i} \leq \mathbf{n}} E\left(\left|X_{\mathbf{i}}\right| I\left[\left|X_{\mathbf{i}}\right|>|\mathbf{n}|^{\alpha_{1}}\right]\right) \\
& \leq \frac{1}{|\mathbf{n}|^{\alpha_{1} r}} \sum_{\mathbf{i} \leq \mathbf{n}} E\left(\left|X_{\mathbf{i}}\right|^{r} I\left[\left|X_{\mathbf{i}}\right|>|\mathbf{n}|^{\alpha_{1}}\right]\right) \\
& \quad \leq \frac{C}{|\mathbf{n}|^{\alpha_{1} r-1}} E|\xi|^{r} \rightarrow 0 \quad \text { as }|\mathbf{n}| \rightarrow \infty,
\end{aligned}
$$

which yields (2.10). Therefore, by (2.12)-(2.16) and Corollary 2.4 we get (2.5).

\section{Competing interests}

The author declares that she has no competing interests.

\section{Author's contributions}

The author read and approved the final manuscript.

\section{Acknowledgements}

The author would like to sincerely thank the anonymous referees for their careful reading of the manuscript and valuable suggestions.

Received: 2 January 2014 Accepted: 15 May 2014 Published: 02 Jun 2014

\section{References}

1. Kuczmaszewska, A, Łagodowski, ZA: Convergence rates in the SLLN for some classes of dependent random fields. J. Math. Anal. Appl. 380, 571-584 (2011)

2. Gut, A: Marcinkiewicz laws and convergence rates in the law of large numbers for random variables with multidimensional indices. Ann. Probab. 6, 469-482 (1978) 
3. Gut, A: Convergence rates for probabilities of moderate deviations for sums of random variables with multidimensional indices. Ann. Probab. 8, 298-313 (1980)

4. Thanh, L: On the strong law of large numbers for $d$-dimensional arrays of random variables. Electron. Commun. Probab. 12, 434-441 (2007)

5. Gut, A, Stadtmüller, U: An asymmetric Marcinkiewicz-Zygmund LLN for random fields. Stat. Probab. Lett. 79, 1016-1020 (2009)

6. Gut, A, Stadtmüller, U: On the Hsu-Robbins-Erdős-Spitzer-Baum-Katz theorem for random fields. J. Math. Anal. Appl. 387, 447-463 (2012)

7. Wichura, MJ: Inequalities with applications to the weak convergence of random processes with multi-dimensional time parameters. Ann. Math. Stat. 60, 681-687 (1969)

10.1186/1029-242X-2014-220

Cite this article as: Ko: On the convergence rates in the asymmetric SLLN for independent and nonidentically distributed random fields. Journal of Inequalities and Applications 2014, 2014:220

Submit your manuscript to a SpringerOpen ${ }^{\circ}$ journal and benefit from:

- Convenient online submission

- Rigorous peer review

- Immediate publication on acceptance

- Open access: articles freely available online

- High visibility within the field

- Retaining the copyright to your article

Submit your next manuscript at $>$ springeropen.com 